\title{
Effects of Planting Density and the Composition of Wheat Cultivar Mixtures on Stripe Rust: An Analysis Taking into Account Limits to the Replication of Controls
}

\author{
K. A. Garrett and C. C. Mundt
}

Department of Botany and Plant Pathology, 2082 Cordley Hall, Oregon State University, Corvallis 97331. Current address of K. A. Garrett: Department of Plant Pathology, Kansas State University, Manhattan 66506. Accepted for publication 11 August 2000.

\begin{abstract}
Garrett, K. A., and Mundt, C. C. 2000. Effects of planting density and the composition of wheat cultivar mixtures on stripe rust: An analysis taking into account limits to the replication of controls. Phytopathology 90: $1313-1321$.

The effect of plant density on disease is not well understood in populations of a single host plant genotype and has been studied even less in mixtures of host genotypes. We performed an experiment to evaluate the effect of wheat planting density on infection by Puccinia striiformis in experimental plots with a single wheat genotype and in plots with two

compared to the weighted mean of disease levels in the corresponding single-genotype plots. The design of the field experiment included limited replication of these reference treatments (that is, there was not a unique pair of single-genotype plots for each mixture plot); therefore, we devised an analysis based on collapsing the data into independent mean observations. Disease reduction due to host diversity was less when one genotype predominated than when both host genotypes were present at nearly equal frequencies. The greatest mean host-diversity effect for reduced disease was at the intermediate planting density of 250 seeds per $\mathrm{m}^{2}$.
\end{abstract} genotypes making up a range of frequencies. Stripe rust severity in single-genotype plots increased with planting density in 1997 but decreased with planting density in 1998. Disease in host mixtures was
Additional keywords: cultivar mixtures, mixture analysis, mixture models, Triticum aestivum.
Although studies of the effects of host genotype diversity on disease are in the early exploratory phase for many host-pathogen systems, much is known about these effects for rusts and mildews of small grains (25). The effect of host diversity on a wheat stripe rust system in Oregon has been studied for over a decade. Greater disease reductions due to host diversity were found for stripe rust than for eyespot of wheat (21). The relative abundance of Puccinia striiformis races changed during the season within mixtures, but races virulent to more than one mixture component did not always come to dominate the pathogen population (8). Host-diversity effects differed for different wheat cultivars and for particular wheat cultivars depending on which other cultivars were mixed with them (18). The relative frequency of wheat genotypes in mixture changed over time (14) and it appears that competition among wheat genotypes may have altered susceptibility to stripe rust (13). The fitness of wheat genotypes was frequency dependent both in the presence and absence of disease (15). Akanda and Mundt (1) found that stripe rust severity on individual cultivars increased approximately linearly with the frequency of a cultivar in mixture. When the size of areas planted to a single genotype was manipulated by planting either random mixtures or alternating rows of genotypes, disease was reduced in both, though the reduction was greater in random mixtures $(4,20)$. Although these aspects of the epidemiology of stripe rust in mixtures have been studied, the effect of planting density has not yet been considered.

Little is known about the influence of planting density on disease in populations of a single host genotype, and even less about the influence of density on disease in populations of multiple host

Corresponding author: K. A. Garrett; E-mail address: kgarrett@ksu.edu

Publication no. P-2000-0918-01R

(C) 2000 The American Phytopathological Society genotypes. Burdon and Chilvers (5), in their review of density effects on plant disease, found a tendency toward increased disease severity at higher densities, though this was by no means a consistent trend. In studies of barley powdery mildew, disease in single-genotype plots decreased with increasing density (12). Barrett and Wolfe (2) observed greater host-diversity effects for reduced powdery mildew at higher planting densities, though this study included single-genotype controls at only one planting density. Pfleeger and Mundt (23) found little evidence for an effect of density on wheat leaf rust in wheat and wild oats mixtures, though they suggested this may have been because of compensatory tillering at low densities. Host-diversity effects might be expected to be greater at higher planting densities because there would be the potential for reduced autoinfection; single plants, and thus single genotypes, make up smaller areas as density increases (22). An alternative hypothesis might be that host-diversity effects would decrease with increasing density because dispersal gradients might become steeper, leading to higher rates of autoinfection. Likewise, if there is an overall increase in disease with higher density, there might be less of a host-diversity effect because there would be a smaller number of generations of pathogen increase before plants approach $100 \%$ disease severity.

For testing whether or not there is a host-diversity effect, the disease severity of a genotype mixture can be compared with the appropriate weighted mean of percent severity in single-genotype plots using linear contrasts. This is a test for whether there is an absolute host-diversity effect (i.e., whether there is a difference between what is observed in mixture and what would be predicted in mixture under the null hypothesis of no host-diversity effect). For comparisons of host-diversity effects under different treatments (e.g., different planting densities), the absolute host-diversity effect may not be a useful measure if the single genotypes vary strongly with treatment. For example, if disease levels are much higher at high densities than at low densities, the absolute 
host-diversity effect may also be larger at high densities as a matter of course. For comparing host-diversity effects at different densities, a measure of the relative host-diversity effect may be more useful. We will refer to the ratio of the observation in mixture to the appropriately weighted mean of observations in singlegenotype plots as the relative mixture response (RMR). Under the null hypothesis of no host-diversity effect, this response would be one. This analysis becomes more complicated than tests using linear contrasts, because the response variable is a ratio and ratios can have undesirable statistical properties (24). In addition, studies of host-diversity effects typically use a limited number of genotypes and then consider several mixtures composed of those genotypes in different combinations and frequencies. Often, there is not a unique set of single-genotype observations for each mixture observation. Any repeated use of observations from singlegenotype plots in the denominators of relative mixture responses will result in observations that are not independent, violating the assumptions of many statistical analyses.

Typical parametric analyses of variance assume that the errors in models are normally, identically, and independently distributed. Such analyses are known to be robust to deviations from assumptions of normality and heteroscedasticity. "To make a preliminary test on variances is rather like putting to sea in a rowing boat to find out whether conditions are sufficiently calm for an ocean liner to leave port" (3). However, deviations from the assumption of independent observations may be more important. Response variables will no longer be independent if observations in the denominator of the RMR are reused within experimental treatments. There are two ways of "reusing" observations for producing response variables that might seem appealing. In the first, referred to as "same standardization for all blocks" by Mead and Riley (19), the mean of all replicates for a particular genotype in a particular treatment would be calculated for the whole field experiment. In our experiment, this would mean taking the mean over all replicates of single-genotype stands of a particular cultivar at a particular density. This estimate would then be used in the denominator of all RMRs for mixtures at that density that include that cultivar. For the second form of reuse, referred to as "separate standardization in each block" by Mead and Riley (19), observations from single-genotype stands are only used within a block. Depending on the experiment, each single-genotype observation might be used only once or more than once for this form of standardization. For both types of reuse, any error associated with the measurements of the reused observations will also be repeated and may make measurements within a treatment appear artificially consistent. This artificially imposed homogeneity within a group may produce an artificial difference between groups or make it appear that an observed difference between groups is defined with more precision than really exists, or both.

Thus, we had three goals in this work. One goal was to estimate the effect of planting density on stripe rust in single-genotype plots. The second was to clarify what artifacts may be introduced by an analysis that ignores a type of dependence structure common in studies of genotype mixtures. The third was to appropriately analyze the impact of mixture components, genotype frequencies, and planting density on the relative mixture response of stripe rust in wheat.

TABLE 1. Susceptibility of wheat cultivars to Puccinia striiformis races used to inoculate experimental plots of two-component cultivar mixtures

\begin{tabular}{lll}
\hline & \multicolumn{2}{c}{ Component susceptible to race } \\
\cline { 2 - 3 } Mixture components & CDL 27 & CDL 29 \\
\hline Faro and Tres & Neither & Faro \\
Faro and Tyee & Tyee & Faro \\
Jacmar and Tyee & Tyee & Jacmar \\
\hline
\end{tabular}

${ }^{a}$ Mixtures with differential susceptibility are those for which each component is susceptible to a different Puccinia striiformis race; $\mathrm{CDL}=$ Cereal Disease Laboratory races.

\section{MATERIALS AND METHODS}

Field study. The field experiment was carried out during two winter wheat seasons, 1996 to 1997 and 1997 to 1998, at the Columbia Basin Agricultural Research Center field station near Pendleton, OR. Field plots, $6.1 \mathrm{~m}$ by 4 rows in size, with $0.36-\mathrm{m}$ row spacing, were planted with four-row strips of the resistant wheat cv. Stephens as a buffer on each side of each plot. Plots were later mowed to $4.9 \mathrm{~m}$ in length. The experiment was planted 15 October 1996 in the first season and 13 to 14 October 1997 in the second.

Four winter club wheat cultivars were included in the study: Jacmar, Tres, Tyee, and a sibling of the commercial cv. Faro, OR 7142 , which has an additional gene for resistance to stripe rust and will here be referred to as Faro. Two races of Puccinia striiformis were used to inoculate the field plots, Cereal Diseases Laboratory (CDL) races 27 and 29; each plot was inoculated with both races on 25 February and 6 March 1997 in the first season and 18 and 24 March 1998 in the second season. The plots were inoculated by introducing infected seedlings of cv. Nugaines, as described in Akanda and Mundt (1). Wheat cultivars were combined to form three different two-component mixtures (Table 1). The components of two mixtures (Faro-Tyee and Jacmar-Tyee) had differential susceptibility (i.e., their components were susceptible to different races of the introduced pathogen population). The other mixture (Faro-Tres) had one component (Tres) that was resistant to both pathogen races. The experiment was originally designed to include a third race, virulent to Tres and avirulent to Faro, but this isolate could not be cultured successfully. Thus, we had two differential mixtures and a comparison between Faro in a differential mixture with Tyee, and Faro in a nondifferential mixture with Tres.

Each of the three wheat genotype combinations was planted at each of four planting densities: 62, 125, 250, and 500 seeds per $\mathrm{m}^{2}$. The 250 seeds per $\mathrm{m}^{2}$ planting density corresponds to the planting rate commonly used by wheat growers at this location. Each genotype combination at each planting density was sown at each of five genotype frequencies: 10/90, 25/75, 50/50, 75/25, and $90 / 10$. The experiment was planted in a randomized block design with four blocks. Each of the treatment combinations described above was represented in each block for a total of 60 mixture plots per block. Each of the four wheat cultivars also appeared as a single-genotype plot at each of the planting densities, adding another 16 plots per block. (If unique single-genotype plots had been included for each mixture plot, there would have been 120 single-genotype plots per block.)

Visual estimates of disease severity (the percentage of leaf area covered by stripe rust lesions on a whole-canopy basis) were made for each experimental plot, and averaged for two observers, on 3 or 4 June 1997 and 31 May or 1 June 1998.

Statistical analysis of single-genotype plots. Disease severity was analyzed as a function of planting density, year, and their interactions. Level of statistical significance of the different factors was determined in an analysis of variance (ANOVA). This and the following statistical analyses were performed using S-Plus (MathSoft Inc., Seattle, WA) and SAS (SAS Institute Inc., Cary, NC) software.

Studies of the statistical properties of analyses using simulated data. To determine how the dependence structure of our data might influence realized $\alpha$ levels, our data structure was analyzed, but with simulated responses free of treatment effects. We performed a simulation study to determine how reusing estimates from single-genotype plots influenced realized $\alpha$ levels. The treatment and replication structure from the experiment was used, but the actual observed disease levels were not. Instead, the simulated percent severity for each plot was simply a constant, one, plus an "error" unique to that plot. Errors were produced by a pseudorandom number generator in S-Plus from a normal distribution with mean 0 and standard deviation $\sigma_{\mathrm{e}}=0.1$. Relative 
mixture responses were then calculated from these simulated "observations". Thus, the new simulated RMRs had the same dependence structure as our real data (before constructing independent means), but there were no treatment effects because all observations were constructed to have the same expectation. Denominators were calculated in the two different ways described by Mead and Riley (19). For the first method, "separate standardization in each block", the same observations from single-genotype plots were reused only within a block. For the second method, "same standardization for all blocks", the mean over blocks of single-genotype plots was used through the experiment. This procedure was repeated 1,000 times, each time with a new set of errors. For each run, an ANOVA was performed on the relative mixture responses and $P$ values for each effect were tallied. In the absence of any treatment effects and under the assumptions of an ANOVA, the $P$ values for any given effect would be uniformly distributed and the probability of a $P$ value falling below 0.05 would be 0.05 . We used the 1,000 simulations to estimate the realized $\alpha$ level for each effect.

To determine how similar, but smaller, data structures might influence realized $\alpha$ levels, we also analyzed a much smaller version of our experimental structure, again with simulated responses free of treatment effects. This was to determine the effect of dependence on realized $\alpha$ levels for smaller experiments in which singlegenotype responses would still be reused, but to a lesser extent. This data structure had two planting densities and two, two-component mixtures with different compositions but one component in common (e.g., Faro-Tres and Faro-Tyee). As in the large data set, each of the cultivars in the smaller data set (Faro, Tres, and Tyee) was replicated only once for each density in each of the four blocks. Thus, each block contained four mixture plots and six plots with single genotypes. As in the larger analysis without unique single-genotype plots for each mixture plot, the same single-genotype result was reused within a density level in calculating the RMR. For 1,000 simulations, the realized $\alpha$ level for an intended $\alpha$ level of 0.05 was recorded.

To determine how nonnormality of the response variables might influence realized $\alpha$ levels, we analyzed our data structure with simulated responses free of treatment effects and additional simulated single-genotype plot responses, so that each relative mixture response was calculated with unique single-genotype plot responses in the denominator. The realized $\alpha$ level for this data set was also measured in 1,000 simulations. The ratio of two normal random variables is not normally distributed and we wished to determine what impact this nonnormality might have on realized $\alpha$ levels. We also wanted to confirm that the method we used to analyze the real data from the field study did not produce undesirable artifacts. To determine whether realized $\alpha$ levels were as desired for the analysis of our independent means data set, described below, we conducted a comparable analysis with simulated responses free of treatment effects. The realized $\alpha$ level for the simulated data was measured for 1,000 simulations in those analyses, as well.

Density, cultivar, and cultivar frequency effects on RMR. Tests of significance based on independent means of RMR were constructed as an alternative to the problematic analysis of dependent data. These tests take advantage of the fact that unique estimates of responses for single-genotype plots are available in each block for each density. Thus, the mean RMR in each density-block combination can be calculated and these means will not share any observations in common from one density-block combination to another. This is the approach used for analyzing the density main effect; the other analyses are based on the same idea, but include fitting of regression lines within densityblock combinations. All of the analyses are described in more technical detail in the appendix. Plots of the residuals versus predicted values from these analyses indicated satisfactory distributions of residuals.
Analysis 1-Planting density main effect. A test of planting density main effects on RMR was constructed by first calculating the mean RMR (averaged over frequencies and genotypes) in each block for each density. This yielded four independent responses per block for a total of 16 independent responses per year. The 2 years were analyzed together in an ANOVA for the linear, quadratic, and cubic portions of a density effect on relative mixture response. Tests of the linear, quadratic, and cubic parts of the density effect were performed using contrasts constructed by Gram-Schmidt orthogonalization (9).

Analysis 2-Genotype frequency main effect and frequency-density interaction. A test of genotype frequency main effects on RMR was constructed by calculating parameter estimates from regression within each density-block combination. Because we expected the mixtures with differential resistance (Faro-Tyee and Jacmar-Tyee) to perform differently than the nondifferential mixture (Faro-Tres), we analyzed the two groups separately. The relative mixture response was fit as a function of the linear and quadratic parts of the genotype frequency effect. The parameter estimates from each density-block combination, which can be assumed to be independent, were then analyzed in a linear model with predictor variables mean, density, and year. The mean response is, itself, a measure of the relationship between genotype frequency and RMR. If the mean is significantly different from 0 , this indicates evidence for a frequency effect. If the density effect is significant, this indicates evidence for a frequency-density interaction. If the year effect is significant, this indicates evidence for a frequency-year interaction. This analysis is similar to the "Standard Two Stage" analysis described by Feldman (11). In the first stage, there is some data dependence, but not in the second stage, where inference is drawn.

Analysis 3-Mixture components main effect and components-density interaction. A test of the mixture components main effect on the RMR was constructed by calculating, within each block-density combination, the mean difference in the relative mixture response between Faro-Tyee and Jacmar-Tyee mixtures and between Faro-Tyee and Faro-Tres mixtures. These two

TABLE 2. Results of analysis of variance for $\log _{10}$-transformed percent stripe rust severity in single-genotype stands of wheat ${ }^{\mathrm{a}}$

\begin{tabular}{|c|c|c|}
\hline Source & df & $P$ value \\
\hline \multicolumn{3}{|l|}{ Years combined } \\
\hline Density & 3 & 0.049 \\
\hline Linear & 1 & 0.019 \\
\hline Quadratic & 1 & 0.183 \\
\hline Cubic & 1 & 0.413 \\
\hline Cultivar & 3 & 0.000 \\
\hline Year & 1 & 0.000 \\
\hline Density-cultivar & 9 & 0.673 \\
\hline Density-year & 3 & 0.000 \\
\hline Cultivar-year & 3 & 0.000 \\
\hline Density-cultivar-year & 9 & 0.225 \\
\hline Residual error & 96 & $\ldots$ \\
\hline \multicolumn{3}{|c|}{ Data from 1997 analyzed separately } \\
\hline Density & 3 & 0.000 \\
\hline Linear & 1 & 0.000 \\
\hline Quadratic & 1 & 0.253 \\
\hline Cubic & 1 & 0.250 \\
\hline Cultivar & 3 & 0.000 \\
\hline Density-cultivar & 9 & 0.199 \\
\hline Residual error & 48 & $\ldots$ \\
\hline \multicolumn{3}{|c|}{ Data from 1998 analyzed separately } \\
\hline Density & 3 & 0.021 \\
\hline Linear & 1 & 0.003 \\
\hline Quadratic & 1 & 0.500 \\
\hline Cubic & 1 & 0.816 \\
\hline Cultivar & 3 & 0.000 \\
\hline Density-cultivar & 9 & 0.990 \\
\hline Residual error & 48 & $\ldots$ \\
\hline
\end{tabular}

${ }^{\text {a }}$ Four densities were considered: 62, 125, 250, and 500 seeds per $\mathrm{m}^{2}$. 
differences were then analyzed in a linear model with predictor variables mean, density, and year. If the mean is significantly different from 0 , this is evidence for a mixture component effect on the RMR. If there is a significant density effect, this is evidence for a component-density interaction. If there is a significant year effect, this is evidence for a component-year interaction.

Analysis 4-Components-frequency interaction and threeway interaction. A test of the mixture components-genotype frequency interaction was constructed by separating the data into groups by block-density-components combinations. Within each group, RMR was fit as a function of the linear and quadratic parts of genotype frequency. Then, within each block-density group, the difference between these parameter estimates for Faro-Tyee mixtures versus Jacmar-Tyee mixtures and for Faro-Tyee mixtures versus Faro-Tres mixtures was calculated. These four differences were analyzed in a linear model with predictors mean, density, and year. If the means are significantly different than 0 , this is evidence for a components-frequency interaction. If there is a significant density effect, this is evidence for a three-way interaction, components-frequency-density. If there is a significant year effect, this is evidence for a significant components-frequencyyear interaction.

\section{RESULTS}

Stripe rust in single-genotype plots increased with density in 1997 and decreased with density in 1998. Residuals showed some tendency to increase in variance with predicted values; therefore, the ANOVA was performed on both untransformed and $\log _{10^{-}}$ transformed response variables. The results for both analyses were quite similar, but results for transformed variables are reported (Table 2). For the analysis of both seasons combined, the effect of density on percent stripe rust severity was statistically significant and the interaction between density and year was highly significant. This interaction reflects the striking difference in response to density between 1997 and 1998 (Fig. 1). When results were analyzed separately by year, the effect of density was highly significant in 1997 and also significant in 1998 (Table 2). The effect of cultivar, year, and their interaction were highly significant, but the interaction between cultivar and density was not (Table 2).

Ignoring the dependence structure in data with limited replication of controls can result in greatly inflated type I error rates. For simulated data without unique single-genotype plots for each mixture plot and with no treatment effects, the type I error rate $(\alpha$ level) was particularly inflated when the mean over blocks was included in the denominator ("same standardization for all blocks") for the calculation of the RMR (Table 3). For this case, the planting density main effect had a realized $\alpha$ level more than 10 times the intended level of 0.05 . That is, $68 \%$ of simulations gave a false positive for the planting density main effect in an analysis which was intended to allow only a standard $5 \%$ level of false positives, had there been no problem with lack of independence. Realized $\alpha$ levels for the mixture composition main effect and some interactions were also greatly inflated. When singlegenotype plot responses from only one block were used in the denominator of the relative mixture response ("separate standardization in each block"), the inflation of realized $\alpha$ levels was not as striking overall, but there was still an important increase for the planting density main effect. Note that the realized $\alpha$ level for the block effect became inflated for this case because observations within a block are dependent. For the simulated data with a simpler treatment structure (Table 3), the inflation of realized $\alpha$ levels was not as dramatic. Still, the realized $\alpha$ level for planting density when the mean over blocks was used in the denominator was more than double the desired level.

In the check of realized $\alpha$ levels for a data set with unique single-genotype plots for each mixture plot and no treatment effects, all $\alpha$ level estimates were within 0.010 of the intended 0.05 level. This indicates that the nonnormality of the ratio response had no important effect on realized $\alpha$ levels for the simulations. In the check of realized $\alpha$ levels for the analysis of independent mean RMRs with responses simulated to include no treatment effects, estimates of the realized $\alpha$ level were within 0.012 of the intended 0.05 level.

The host-diversity effect for reduced disease was greatest at the intermediate density (results of analysis 1). In the analysis of independent means, only the quadratic part of the density effect on the relative mixture response was statistically significant (Table 4). There was some evidence for a difference between years, but none for a density-year interaction. The results are portrayed separately by year (Fig. 2) to show the striking similarity in response between the two seasons.

For the two differential mixtures, disease reduction was greatest when the two components were present at intermediate frequency (results of analysis 2). This result was highly significant, though the effect of frequency was not significant for
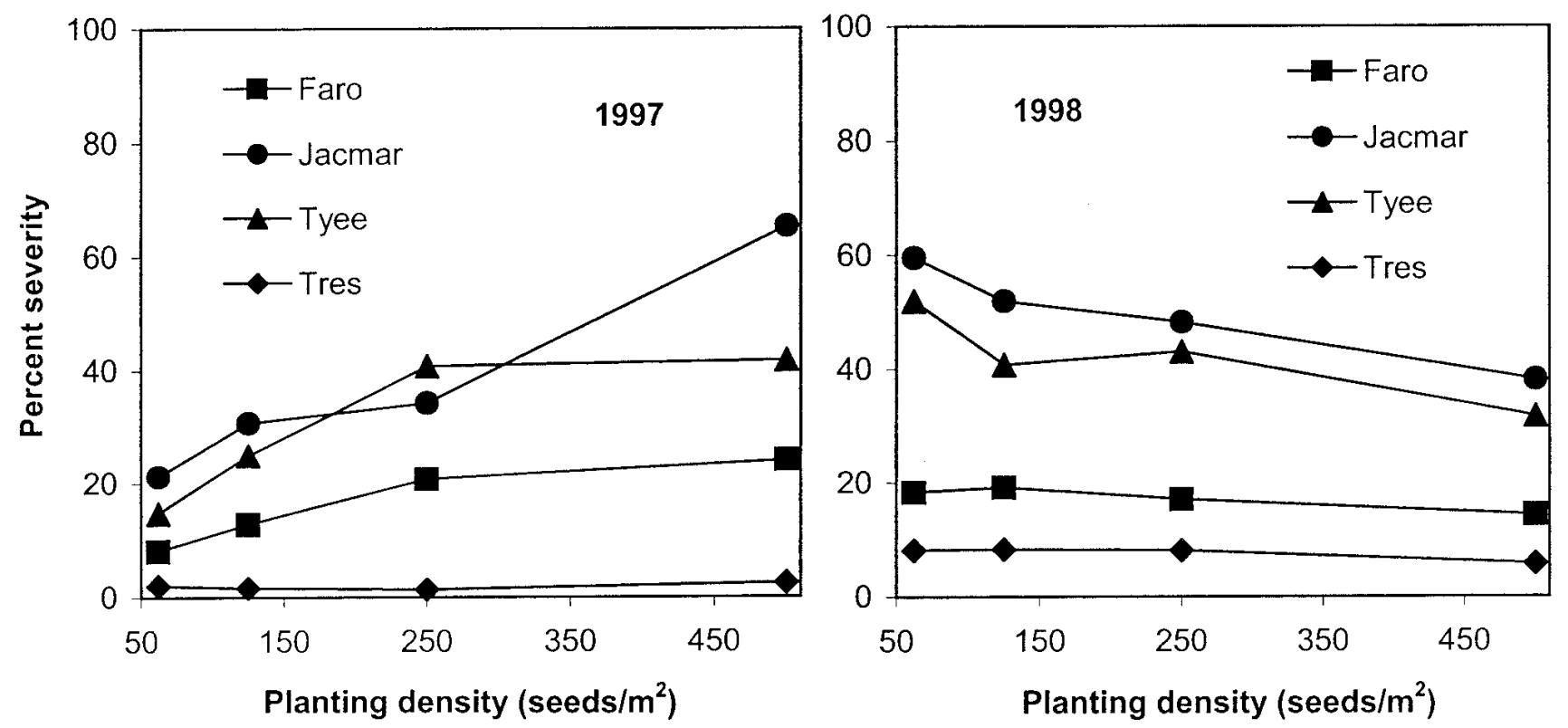

Fig. 1. Percent severity of stripe rust in single-genotype wheat plots as a function of wheat planting density. 
the nondifferential mixture (Faro-Tres). None of the interactions in this analysis were highly significant, though there was some evidence for an interaction between density and frequency for the nondifferential mixture (Fig. 3; Table 5).

The host-diversity effect for reduced disease was greater in Faro-Tyee mixtures than in Faro-Tres mixtures (results of analysis 3). This difference was large and statistically significant (Figs. 3 and 4; Table 6). There were some estimated differences in interactions between composition and density (Fig. 4), but this interaction was not significant (Table 6). Likewise, there was no evidence for a difference between Jacmar-Tyee and Faro-Tyee mixtures (Table 6).

There was evidence for a composition-frequency-density interaction for the comparison of Faro-Tres and Faro-Tyee (results of analysis 4). The quadratic and cubic parts of density appeared to be the main contributors to this effect (Table 7). There was also some evidence for a composition-frequency-year interaction for that comparison. None of the interactions for the FaroTyee and Jacmar-Tyee comparison were statistically significant.

\section{DISCUSSION}

For the single genotypes, density effects on percent severity were reversed from one year to another. We can speculate that part of this difference might be due to the fact that there was greater tillering in 1998 than in 1997. Compensation from increased tillering at low densities can prevent treatments from representing as wide a range of densities as planned. For planted rates of 62,125 , 250 , and 500 seeds $\mathrm{m}^{-2}$, realized tiller densities averaged 157,218 , 274, and 329 tillers $\mathrm{m}^{-2}$ in 1997 and 270, 308, 369, and 395 tillers $\mathrm{m}^{-2}$ in 1998 (J. Brunet and C. C. Mundt, unpublished data). The general tendency for disease levels to increase with density (5) would be more likely to hold in 1997, when the low levels of tillering made differences between density treatments more striking. However, this does not explain the reverse linear trend in 1998, which was statistically significant when the years were analyzed separately (Table 2). Different levels of nutrition and water stress experienced by plants in the two years might have influenced susceptibility.

Our field study consisted of 304 experimental plots each year and was only part of a larger study. One way of addressing the problem of dependent data would have been to include unique

TABLE 3. Realized $\alpha$ levels (Type I error rates) from analysis of relative mixture response using simulated data without unique single-genotype plots for each genotype-mixture plot $^{\mathrm{a}}$

\begin{tabular}{lccc}
\hline & & \multicolumn{2}{c}{ Realized $\alpha$ level } \\
Effect & df & Same $^{\mathrm{b}}$ & Separate $^{\mathrm{c}}$ \\
\hline Experimental design of field experiment & & & \\
$\quad$ Block & 3 & 0.05 & 0.481 \\
A = genotype pair & 2 & 0.33 & 0.136 \\
B = genotype frequency & 2 & 0.16 & 0.034 \\
C = planting density & 3 & 0.68 & 0.480 \\
A-B & 4 & 0.11 & 0.007 \\
A-C & 6 & 0.54 & 0.262 \\
B-C & 6 & 0.21 & 0.033 \\
A-B-C & 12 & 0.13 & 0.002 \\
Smaller design for comparison & & & \\
Block & 3 & 0.056 & 0.081 \\
A = genotype pair & 1 & 0.075 & 0.045 \\
B = planting density & 1 & 0.129 & 0.070 \\
B-C & 1 & 0.069 & 0.047 \\
\hline
\end{tabular}

a Simulations included no treatment effects and were for one season's data for intended level $\alpha=0.05\left(\sigma_{\mathrm{e}}=0.1\right)$. This analysis was performed to determine the magnitude of artifacts that might be produced when data dependence was not taken into account in performing such analyses.

${ }^{\mathrm{b}}$ Means for single-genotype plots were calculated over the whole experiment and were the same for each block.

${ }^{\mathrm{c}}$ Means for single-genotype plots were calculated separately for each block. single-genotype plots paired with each mixture plot. This would be similar to a paired plot design, except that each mixture plot would have two corresponding single-genotype plots. If the current study had included such unique plots, there would have been an unrealistic total of 720 experimental plots. Thus, we were forced to confront the problem of dependent data in our analyses of the RMR.

Reuse of observations from single cultivar plots in calculating RMRs did inflate realized $\alpha$ levels, demonstrating that use of the RMR without accounting for possible dependence structures can produce misleading results. However, our field study was unusual in the large number of treatments and the large number of times the response from each single-genotype plot could have been reused. In our simulation of a smaller experiment, inflation of $\alpha$ levels was less of a problem. The method we used in our analysis of the field data, analyzing independent mean responses, gets around the problem, but at the cost of reduced statistical power. There is reduced power because the unique observations of percent severity in mixture plots are essentially averaged together in producing the mean RMRs. Greater replication of single-genotype plots short of unique single-genotype plots for each mixture plot could increase the power of the experiment by giving a greater number of independent mean RMRs.

TABLE 4. Analysis of variance on the relative mixture response (RMR) for stripe rust percent severity in wheat genotype mixtures ${ }^{\mathrm{a}}$

\begin{tabular}{lcc}
\hline Source & df & $P$ value \\
\hline Density & 3 & 0.099 \\
Linear & 1 & 0.360 \\
Quadratic & 1 & 0.021 \\
Cubic & 1 & 0.803 \\
Year & 1 & 0.162 \\
Density-year & 3 & 0.892 \\
Residual & 24 & $\ldots$ \\
\hline
\end{tabular}

a RMR is the ratio with numerator equal to the percent severity of wheat stripe rust in a mixture of wheat genotypes and denominator equal to the weighted average of the percent severity in single-genotype plots of the mixture components. These results are from the analysis of independent means described in the appendix, analysis 1.

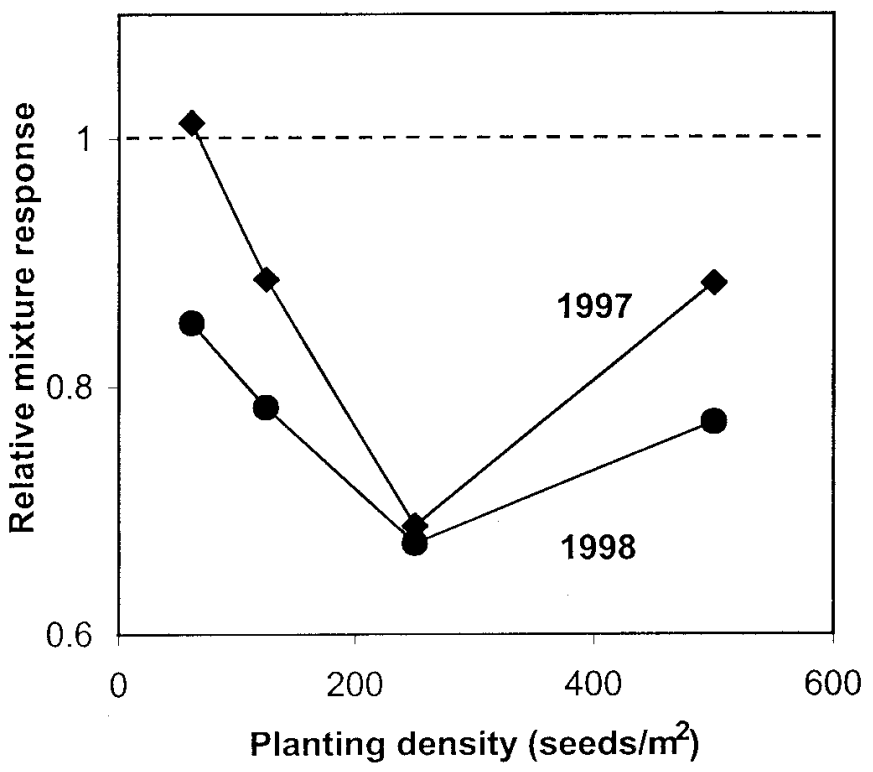

Fig. 2. Mean relative mixture response (RMR) for wheat stripe rust as a function of planting density (corresponding to analysis 1). The RMR is the ratio with the percent severity of wheat stripe rust in a mixture of wheat genotypes in the numerator and the weighted average of the percent severity in single-genotype plots of the mixture components in the denominator. The line at $\mathrm{RMR}=1$ indicates the result under the null hypothesis of no host diversity effect on percent severity. 
Federer (10) has discussed statistical issues involved in analyzing land equivalent ratios (LERs) in studies of intercropping. The LER is "a measure of the efficiency of an intercrop in terms of land area required under sole cropping to give the yields obtained from the individual crops" (10). This may be expressed as a ratio similar to the RMR. Federer (10) recommended use of known response levels for single-genotype plots that could be treated as constants in constructing the denominator of the LER. This would be a good choice if constants were available, but, in general, the response in single-genotype plots must be estimated under environmental conditions similar to the conditions for the mixture plots. Mean responses from single-genotype plots might approximate a known constant response, but, unless there are enough replicates to produce a very low variance for the estimate, the potential for artifacts that was illustrated in our simulations will still be present.

For our experiment, there was adequate power to detect effects of interest using the method of collapsing the data set to independent means. Alternative methods of analysis might be promising for some studies that lack sufficiently large sets of independent observations. Randomization tests might be used with the incorporation of randomization of residuals, for data sets with appropriately distributed residuals. It may prove useful to con- struct a parametric ANOVA without the assumption of independent observations by directly specifying the correlation between observations (17). It may also be possible to modify mixture models for this purpose (7), though the emphasis in mixture models has been on absolute effects. In standard mixture models, the responses in single-genotype plots would function similarly to covariates. For these analyses it would still be necessary to carefully consider how observations are reused, because reusing single-genotype observations as predictor variables within a treatment could lead to problems with colinearity (9). Applying a series of linear contrasts to log-transformed observations of percent severity might give results somewhat similar to the comparisons of RMR reported here, because the log of a ratio is equal to the difference between logs. The results would not be the same, however, because the contrasts would be based on the mean of logs rather than the log of means. Tests for the presence of host-diversity effects using such an approach might be undesirably conservative.

Using the conservative analysis based on independent means, average density effects on relative mixture responses were consistently quadratic over two very different seasons. This response was unexpected. It is possible that a trend for increased host-diversity effects with increasing density (because of smaller geno-
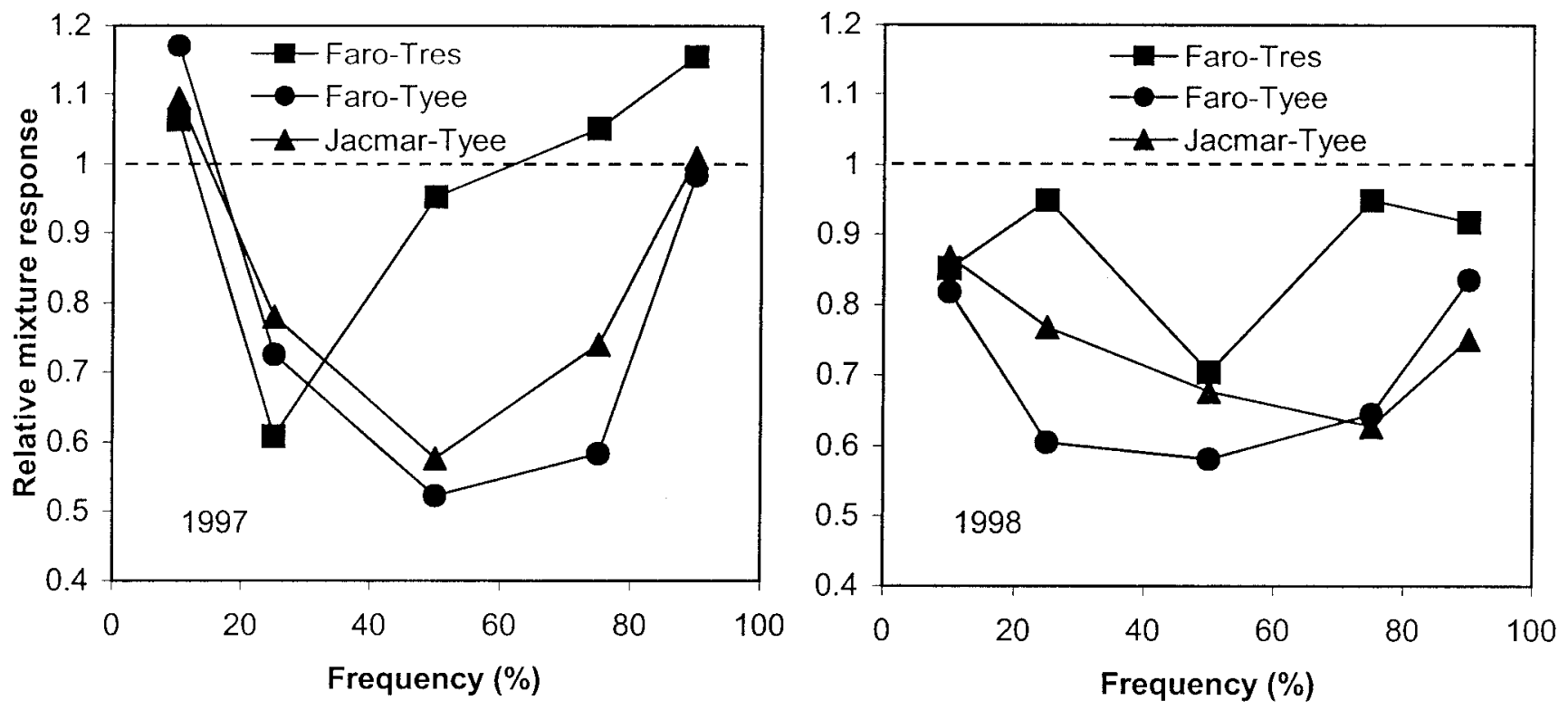

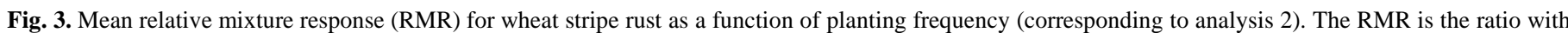

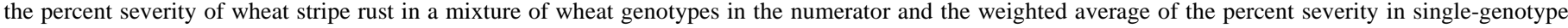

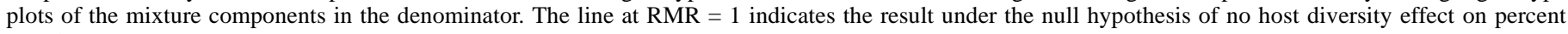
severity.

TABLE 5. Analysis of variance on the relative mixture response (RMR) for stripe rust percent severity in wheat genotype mixtures ${ }^{\mathrm{a}}$

\begin{tabular}{|c|c|c|c|c|c|}
\hline \multirow[b]{3}{*}{ Source } & \multirow[b]{3}{*}{ df } & \multicolumn{4}{|c|}{$P$ values } \\
\hline & & \multicolumn{2}{|c|}{ Differential mixtures } & \multicolumn{2}{|c|}{ Nondifferential mixture } \\
\hline & & Linear & Quadratic & Linear & Quadratic \\
\hline Genotype frequency $(\mathrm{GF})^{\mathrm{b}}$ & 1 & 0.005 & 0.002 & 0.451 & 0.540 \\
\hline GF-density ${ }^{c}$ & 3 & 0.549 & 0.615 & 0.095 & 0.099 \\
\hline GF-yeard & 1 & 0.474 & 0.349 & 0.310 & 0.312 \\
\hline GF-density-year ${ }^{\mathrm{e}}$ & 3 & 0.520 & 0.485 & 0.738 & 0.826 \\
\hline Residual & 24 & $\ldots$ & $\ldots$ & $\ldots$ & $\ldots$ \\
\hline $\begin{array}{l}\text { a RMR is the ratio with nut } \\
\text { average of the percent sev } \\
\text { appendix, analysis } 2 \text {. } \\
\text { b Corresponds to test of mea } \\
\text { c Corresponds to test of den } \\
\text { d Corresponds to test of yea } \\
\text { e Corresponds to test of den }\end{array}$ & $\begin{array}{l}\text { to th } \\
\text { geno } \\
\text { n ana } \\
\text { s } 2 \text {. }\end{array}$ & $\begin{array}{l}\text { ity of wh } \\
\text { e mixtur }\end{array}$ & $\begin{array}{l}\text { n a mixture } \\
\text { hese results }\end{array}$ & $\begin{array}{l}\text { ypes and } \\
\text { alysis of }\end{array}$ & $\begin{array}{l}\text { ual to the } \\
\text { ans describe }\end{array}$ \\
\hline
\end{tabular}


type unit; 22) and a trend for decreased host-diversity effects with increasing density (because of shortened dispersal gradients) combined to produce an optimum at the intermediate density. Fortunately for potential users of wheat mixtures, this optimum was at the density typical of wheat production systems in the study area.

As in Akanda and Mundt's (1) study, the effects of host diversity in differential mixtures were usually greatest when the frequency of each component was minimized. For mixtures composed of only two genotypes, this would be the 50:50 genotype proportion. The results for the nondifferential Faro-Tres mixture were more erratic. Mixture theory would argue that mixtures with the lowest frequency of the susceptible Faro would experience the greatest mixture effect for reduced disease (16). But we would also expect high variability in disease severity for the Faro-Tres mixtures with a low frequency of Faro because only a small proportion of plants can have appreciable infection. Also, in the analysis of independent means, tests for this nondifferential mixture are based on averages over only half as many observations in mixtures as for the differential mixtures.

Differential mixtures can experience a greater mixture effect for reduced disease because each component can benefit from reduced inoculum. There is also the potential for benefits from induced resistance (6). In nondifferential mixtures, only the more susceptible component benefits. As might be expected, the host- diversity effect for reduced disease was greater for the mixture with differential susceptibility (Faro-Tyee) than for the mixture with varying susceptibility (Faro-Tres). But this comparison is based on only one nondifferential mixture and differences due to differentiality are confounded with other ways in which Tyee and Tres differ in mixture with Faro.

There was some evidence for interactions with density, so we should emphasize that our results in the analysis of density main effects (analysis 1) are average effects. The RMR will vary throughout an epidemic, often reaching a maximum at an intermediate time point (26). Disease levels could not be estimated at exactly the same point in both seasons; this difference in timing may explain some differences between years and interactions between year and treatment or density. Changes in cultivar frequency over time may also have caused some differences in hostdiversity effects between treatments. Seed from the first season was used to plant the second season; therefore, changes in cultivar frequency could accumulate over two seasons. Between the original planting in 1996 and the planting in 1997, frequencies had changed by an average of $-3 \%$ for Faro in Faro-Tres mixtures, $+2 \%$ for Faro in Faro-Tyee mixtures, and $-12 \%$ for Jacmar in Jacmar-Tyee mixtures (J. Brunet and C. C. Mundt, unpublished data). Strongly significant interactions between frequency and density were not found in this experiment.
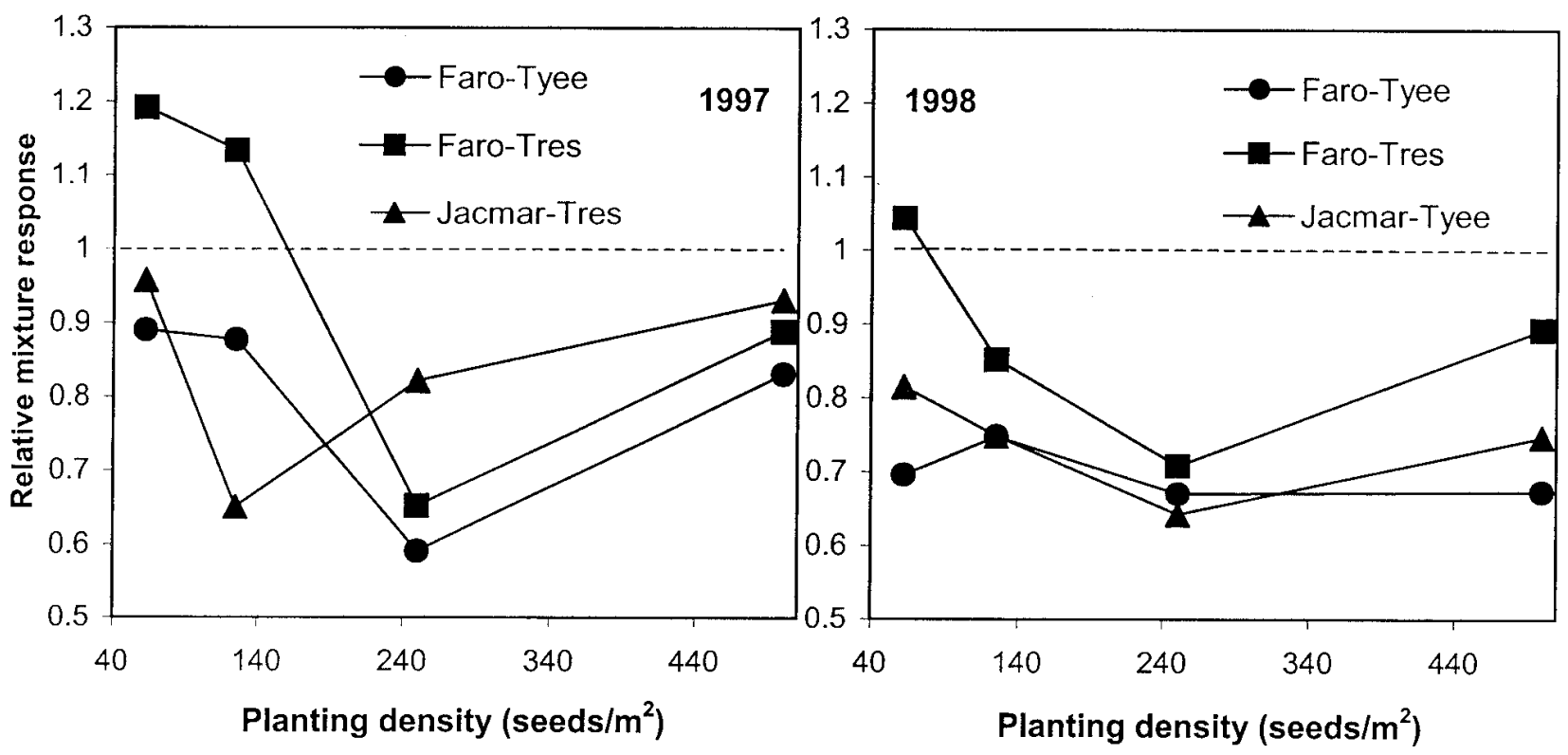

Fig. 4. Mean relative mixture response (RMR) for wheat stripe rust as a function of planting density (corresponding to analysis 3). The RMR is the ratio with the percent severity of wheat stripe rust in a mixture of wheat genotypes in the numerator and the weighted average of the percent severity in single-genotype plots of the mixture components in the denominator. The line at RMR $=1$ indicates the result under the null hypothesis of no host diversity effect on percent severity.

TABLE 6. Analysis of variance on the relative mixture response (RMR) for stripe rust percent severity in wheat genotype mixtures ${ }^{\mathrm{a}}$

\begin{tabular}{lccc}
\hline & & & \multicolumn{2}{c}{$P$ values } \\
\cline { 3 - 4 } Source & df & Faro-Tres versus Faro-Tyee & Faro-Tyee versus Jacmar-Tyee \\
\hline Genotype composition (GC) $^{\mathrm{b}}$ & 1 & 0.044 & 0.393 \\
GC-densityc $^{\mathrm{c}}$ GC-year & 3 & 0.690 & 0.353 \\
GC-density-year $^{\mathrm{e}}$ & 1 & 0.960 & 0.987 \\
Residual & 3 & 0.920 & 0.377 \\
\end{tabular}

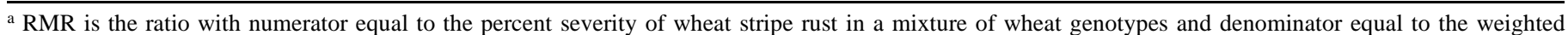
average of the percent severity in single-genotype plots of the mixture components. These results are from the analysis of independent means described in the appendix, analysis 3 .

b Corresponds to test of mean (intercept) in analysis 3 .

c Corresponds to test of density in analysis 3 .

${ }^{\mathrm{d}}$ Corresponds to test of year in analysis 3 .

e Corresponds to test of year-density in analysis 3 . 
To summarize, the effects of density on stripe rust severity were reversed in the 2 years of our study. Despite this dramatic difference between the 2 years, the average effect of density on the RMR was consistently quadratic in the 2 years. The greatest hostdiversity effect in both years was for the density typically planted by growers in the area of the study. This strong influence of planting density on host-diversity effects suggests that caution should be used when applying the results of one mixture study to systems that might involve different planting densities. In addition, analyses of the RMR should be designed to take into account which observations are independent.

\section{APPENDIX}

Formulation of tests on data analyzed in the form of independent means. The percent severity was observed in each plot and notated as $y_{i j l t}$ within a single-genotype plot and $y_{i j k(1, m) t}$ within a genotype mixture for the $i^{t h}$ block within year $t(i=$ $1, \ldots, 4 ; t=97,98)$, the $j^{\text {th }}$ planting density $(j=1, \ldots, 4)$, the $k^{\text {th }}$ genotype frequency $\left(k=1, \ldots .5 ; p_{1}=0.10, p_{2}=0.25, p_{3}=0.50\right.$, $\left.p_{4}=0.75, p_{5}=0.90\right)$, and the $1^{\text {th }}\left(\right.$ and $m^{\text {th }}$ ) genotype (pairs are $F R$, $F Y$, and $J Y$ ). The relative mixture response was calculated for each mixture plot as

$$
R_{i j k(1, m) t}=y_{i j k(1, m) t} /\left[p_{k} \times y_{i j \mathrm{j} t}+\left(1-p_{k}\right) y_{i j m t}\right]
$$

where $p_{k}$ is the $k^{\text {th }}$ frequency.

Analysis 1-Planting density. In this analysis, the mean of $R_{i j k(1, m) t}$ was calculated for each block $\times$ density $\times$ year combination and notated as $\bar{R}_{i j . t .}$. The model we fit was

$$
\bar{R}_{i j . t}=\mu+\delta_{j}+\gamma_{t}+(\delta \gamma)_{j t}+\varepsilon_{i j t}
$$

where $\delta_{j}$ corresponds to the $j^{\text {th }}$ density.

Analysis 2-Genotype frequency. Because we would expect frequency to influence differential and nondifferential mixtures differently, we analyzed them separately. In the first stage of the analysis, the models

$$
\bar{R}_{i j k . t}=\beta_{0 i j t}+\beta_{1 i j t} p_{k}+\beta_{2 i j t} p_{k}^{2}+\varepsilon_{i j k t}
$$

where $p_{k}$ is the $k^{\text {th }}$ genotype frequency, were fit for each combination of ijt. In the next stage of the analysis, a multivariate response model was fit as

$$
\left[\begin{array}{l}
\beta_{1 i j t} \\
\beta_{2 i j t}
\end{array}\right]=\mu+\delta_{j}+\gamma_{t}+(\delta \gamma)_{j t}+\varepsilon_{i j t}
$$

where $\mu$ corresponds to the test for frequency main effects, $\delta_{j}$ corresponds to the test for density-frequency interactions, $\gamma_{t}$ corresponds to the test for frequency-year interactions and $(\delta \gamma)_{j t}$ corresponds to the test for density-frequency-year interactions.

Analysis 3-Mixture components main effect and components-density interaction. In the first stage of the analysis, the differences between

$$
\begin{aligned}
D_{i j F t} & =\bar{R}_{i j .(F R) t}-\bar{R}_{i j .(F Y) t} \\
D_{i j Y t} & =\bar{R}_{i j .(F Y) t}-\bar{R}_{i j .(J Y) t}
\end{aligned}
$$

were calculated for each combination of ijt. In the next stage of the analysis a multivariate response model was fit as

$$
\left[\begin{array}{c}
D_{i j F t} \\
D_{i j Y t}
\end{array}\right]=\mu+\delta_{j}+\gamma_{t}+(\delta \gamma)_{j t}+\varepsilon_{i j t}
$$

where $\mu$ corresponds to the test for mixture components main effects, $\delta_{j}$ corresponds to the test for density-components interactions, $\gamma_{t}$ corresponds to the test for components-year interactions and $(\delta \gamma)_{j t}$ corresponds to the test for density-componentsyear interactions.

Analysis 4-Components-frequency interaction and threeway interaction. In the first stage of the analysis, the models

$$
\bar{R}_{i j k(1, m) t}=\beta_{0 i j(1, m) t}+\beta_{1 i j(1, m) t} p_{k}+\beta_{2 i j(1, m) t} p_{k}^{2}
$$

where $p_{k}$ is the $k_{t h}$ genotype frequency, were fit for each combination of $i j(1, m) t$. In the next stage of the analysis, the differences

$$
\begin{aligned}
D_{1 i j F t} & =\beta_{1 i j(F R) t}-\beta_{1 i j(F Y) t} \\
D_{1 i j Y t} & =\beta_{1 i j(F Y) t}-\beta_{1 i j(J Y) t} \\
D_{2 i j F t} & =\beta_{2 i j(F R) t}-\beta_{2 i j(F Y) t} \\
D_{2 i j Y t} & =\beta_{2 i j(F Y) t}-\beta_{2 i j(J Y) t}
\end{aligned}
$$

\begin{tabular}{|c|c|c|c|c|c|}
\hline \multirow[b]{3}{*}{ Source ${ }^{b}$} & \multirow[b]{3}{*}{$\mathrm{df}$} & \multicolumn{4}{|c|}{$P$ values } \\
\hline & & \multicolumn{2}{|c|}{ Faro-Tres versus Faro-Tyee } & \multicolumn{2}{|c|}{ Faro-Tyee versus Jacmar-Tyee } \\
\hline & & Linear & Quadratic & Linear & Quadratic \\
\hline GC-GF-density ${ }^{\mathrm{d}}$ & 3 & 0.099 & 0.102 & 0.506 & 0.449 \\
\hline Linear density & 1 & 0.926 & 0.829 & $\ldots$ & $\ldots$ \\
\hline Quadratic density & 1 & 0.102 & 0.110 & $\ldots$ & $\ldots$ \\
\hline Cubic density & 1 & 0.054 & 0.054 & $\ldots$ & $\ldots$ \\
\hline Residual & 27 & $\ldots$ & $\ldots$ & $\ldots$ & $\ldots$ \\
\hline
\end{tabular}

were calculated for each combination of ijt. In the third stage of the analysis, a multivariate response model was fit as

$$
\left[\begin{array}{c}
D_{1 i j F t} \\
D_{1 i j Y t} \\
D_{2 i j F t} \\
D_{2 i j Y t}
\end{array}\right]=\mu+\delta_{j t}+\gamma_{t}+(\delta \gamma)_{j t}+\varepsilon_{i j t}
$$

where $\mu$ corresponds to the test for a components-frequency interaction, $\delta_{j}$ corresponds to the test for a density-componentsfrequency interaction, $\gamma_{t}$ corresponds to the test for a componentsfrequency-year interaction and $(\delta \gamma)_{j t}$ corresponds to the test for a density-components-frequency-year interaction.

TABLE 7. Analysis on the relative mixture response (RMR) for stripe rust percent severity in wheat genotype mixtures ${ }^{\mathrm{a}}$

${ }^{a}$ RMR is the ratio with numerator equal to the percent severity of wheat stripe rust in a mixture of wheat genotypes and denominator equal to the weighted average of the percent severity in single-genotype plots of the mixture components. These results are from the analysis of independent means described in the appendix, analysis 4 .

${ }^{\mathrm{b}} \mathrm{GC}=$ genotype composition and $\mathrm{GF}=$ genotype frequency.

${ }^{\mathrm{c}}$ Corresponds to test of mean (intercept) in analysis 4.

${ }^{\mathrm{d}}$ Corresponds to test of genotype frequency-density interaction in analysis 4.

${ }^{\mathrm{e}}$ Corresponds to test of genotype frequency-year interaction in analysis 4.

${ }^{\mathrm{f}}$ Corresponds to test of genotype frequency-year-density interaction in analysis 4. 


\section{ACKNOWLEDGMENTS}

This work was funded in part by USDA NRI grant 96-35303-3206 to C. C. Mundt and J. Brunet. We thank J. Brunet, K. B. Johnson, B. F. J. Manly, P. A. Murtaugh, C. B. Pereira, Phytopathology reviewers, and especially D. S. Birkes for comments that improved this work; and L. W. Cohnstaedt, D. J. Coyle, S. DeMay, M. E. Hoffer, R. Lau, and N. J. Rodecap for help realizing the field study.

\section{LITERATURE CITED}

1. Akanda, S. I., and Mundt, C. C. 1996. Effects of two-component wheat cultivar mixtures on stripe rust severity. Phytopathology 86:347-353.

2. Barrett, J. A., and Wolfe, M. S. 1980. Pathogen response to host resistance and its implication in breeding programs. EPPO Bull. 10:341-347.

3. Box, G. E. P. 1953. Non-normality and tests on variances. Biometrika 40:333.

4. Brophy, L. S., and Mundt, C. C. 1991. Influence of plant spatial patterns on disease dynamics, grain yield and plant competition in genetically diverse wheat populations. Agric. Ecosyst. Environ. 35:1-12.

5. Burdon, J. J., and Chilvers, G. A. 1982. Host density as a factor in plant disease ecology. Annu. Rev. Phytopathol. 20:143-166.

6. Calonnec, A., Goyeau, H., and de Vallavieille-Pope, C. 1996. Effects of induced resistance on infection efficiency and sporulation of Puccinia striiformis on seedlings in varietal mixtures and on field epidemics in pure stands. Eur. J. Plant Pathol. 102:733-741.

7. Cornell, J. A. 1990. Experiments with Mixtures: Designs, Models, and the Analysis of Mixture Data. 2nd ed. Wiley-Interscience, New York.

8. DiLeone, J. A., and Mundt, C. C. 1994. Effect of wheat cultivar mixtures on populations of Puccinia striiformis races. Plant Pathol. 43:917-930.

9. Draper, N. R., and Smith, H. 1998. Applied Regression Analysis. 3rd ed. Wiley-Interscience, New York.

10. Federer, W. T. 1993. Statistical design and analysis for intercropping experiments, vol. 1. Two Crops. Springer-Verlag, New York.

11. Feldman, H. A. 1988. Families of lines: Random effects in linear regression analysis. J. Appl. Physiol. 64:1721-1732.

12. Finckh, M. R., Gacek, E. S., Czembor, H. J., and Wolfe, M. S. 1999.
Host frequency and density effects on disease and yield in mixtures of barley. Plant Pathol. 48:807-816.

13. Finckh, M. R., and Mundt, C. C. 1992. Plant competition and disease in genetically diverse wheat populations. Oecologia 91:81-92.

14. Finckh, M. R., and Mundt, C. C. 1992. Stripe rust, yield, and plant competition in wheat cultivar mixtures. Phytopathology 82:905-913.

15. Finckh, M. R., and Mundt, C. C. 1993. Effects of stripe rust on the evolution of genetically diverse wheat populations. Theor. Appl. Genet. 85:809-821.

16. Garrett, K. A., and Mundt, C. C. 1999. Epidemiology in mixed host populations. Phytopathology 89:984-990.

17. Geisser, S., and Greenhouse, S. W. 1958. An extension of Box's results on the use of the $F$-distribution in multivariate analysis. Ann. Math. Stat. 29:885-891.

18. Knott, E. A., and Mundt, C. C. 1990. Combining ability analysis of wheat cultivar mixtures under diseased and non-diseased conditions. Theor. Appl. Genet. 80:313-320.

19. Mead, R., and Riley, J. 1981. A review of statistical ideas relevant to intercropping research. J. Res. Stat. Soc. A 144:462-509.

20. Mundt, C. C., Brophy, L. S., and Kolar, S. C. 1996. Effect of genotype unit number and spatial arrangement on severity of yellow rust in wheat cultivar mixtures. Plant Pathol. 45:215-222.

21. Mundt, C. C., Brophy, L. S., and Schmitt, M. E. 1995. Disease severity and yield of pure-line wheat cultivars and mixtures in the presence of eyespot, yellow rust, and their combination. Plant Pathol. 44:173-182.

22. Mundt, C. C., and Leonard, K. J. 1986. Analysis of factors affecting disease increase and spread in mixtures of immune and susceptible plants in computer-simulated epidemics. Phytopathology 76:832-840.

23. Pfleeger, T. G., and Mundt, C. C. 1998. Wheat leaf rust severity as affected by plant density and species proportion in simple communities of wheat and wild oats. Phytopathology 88:708-714.

24. Sokal, R. R., and Rohlf, F. J. 1994. Biometry: The Principles and Practice of Statistics in Biological Research. W. H. Freeman \& Co., New York.

25. Wolfe, M. S. 1985. The current status and prospects of multiline cultivars and variety mixtures for disease resistance. Annu. Rev. Phytopathol. 23:251-273.

26. Wolfe, M. S., and Barrett, J. A. 1980. Can we lead the pathogen astray? Plant Dis. 64:148-155. 